\title{
MYXOMYCETES EM PALMEIRAS (ARECACEAE)
}

\author{
Maria Helena Alves ${ }^{1}$ \\ Recebido em 07.07.94. Aceito em 10.01.95. \\ Laise de Holanda Cavalcanti ${ }^{2}$
}

RESUMO - (Myxomycetes em Palmeiras (Arecaceae). As palmeiras oferecem um ecossistema especializado que abriga organismos variados, incluindo Myxomycetes. Este trabalho relata doze espécies de Myxomycetes encontradas em folhas mortas, espata e estipe de Acrocomia intumescens Drude (macaúba), Copernicea prunifera (Mill.) H. E. Moore (carnaúba), Mauritia flexuosa Mart. (buriti) e Orbygnia phalerata Mart. (babaçu). Constitui-se área de coleta o Município do Crato, Ceará, Nordeste do Brasil ( $7^{\circ} 30^{\prime} 00^{\prime \prime} \mathrm{S}, 39^{\circ} 00^{\prime} 00^{\prime \prime} \mathrm{W}, 400-1200 \mathrm{~m}$ de altitude). Coletas aleatórias foram efetuadas e as frutificações foram pesquisadas em órgãos vivos ou mortos das palmeiras. Exsicatas foram depositadas no Herbário UFP (Departamento de Botânica da Universidade Federal de Pernambuco, Recife - PE, Brasil). As espécies registradas pertencem aos gêneros Ceratiomyxa (1), Dictydium (1), Arcyria (1), Hemitrichia (1), Badhamia (1), Fuligo (1), Physarum (3), Stemonitis (2) e Comatricha (1). Ceratiomyxa fruticulosa (Müll.) Macbr., Physarum stellatum (Mass.) Mart., Stemonitis pallida Win. e Comatricha typhoides (Bull.) Rost. são referidas pela primeira vez para este tipo de substrato. Este trabalho eleva para 35 o número de espécies de Myxomycetes assinaladas sobre palmeiras até o momento.

Palavras-chave: Myxomycetes, substrato, Arecaceae, Ceará, Brasil.

\begin{abstract}
Myxomycetes on palm trees (Arecaceae). Palm trees are specialized ecossystems which hosts a wide variety of organisms, including Myxomycetes. This paper reports twelve species of Myxomycetes found on dead leaves, spathes and stipitis of Acrocomia intumescens Drude, Copernicea prunifera (Mill.) H. E. Moore, Mauritia flexuosa Mart., and Orbygnia phalerata Mart. The collecting area is located in the Municipality Crato of Ceara State, Northeastern Brazil ( $7^{\circ} 30^{\prime} 00^{\prime \prime} \mathrm{S}, 39^{\circ} 00^{\prime} 00^{\prime \prime} \mathrm{W}, 400-1200 \mathrm{~m}$ altitude). Samples were taken at random and Myxomycetes fructifications were searched on dead and living organs of palm trees. Exsiccatae have been deposited in the Herbarium UFP (Department of Botany, Federal University of Pernambuco, Recife-PE, Brazil). The species registered belong to the genera Ceratiomyxa (1), Dictydium (1), Arcyria (1), Hemitrichia (1), Badhamia (1), Fuligo (1), Physarum (3), Stemonitis (2) and Comatricha (1). Ceratiomyxa fruticulosa (Müll.) Macbr., Stemonitis pallida Win, Comatricha typhoides (Bull.) Rost. and Physarum stellatum (Mass.) Mart. are reported for the first time on this substrate. This study raises to 35 the number of Myxomycetes species occurring on palm trees substrates.
\end{abstract} 1 Universidade Federal de Pernambuco (UFPE), Centro de Ciências Biológicas, Mestrado em
Criptógamos.

2 UFPE - Centro de Ciências Biológicas, Departamento de Botânica, Recife-PE. 
Key words: Myxomycetes, substrate. Arecaceae, Ceará State. Brazil.

\section{Introdução}

Os primeiros registros de ocorrência de Myxomycetes no Brasil datam do século passado, referentes às Regiões Norte e Sul do país (Cavalcanti, 1974). Para a região Nordeste já foram referidas mais de 100 espécies, em localidades próximas ao litoral, nos Estados da Bahia (Torrend 1915; Gottsberger 1968), Alagoas (Cavalcanti et al. 1985, Santos et al. 1986), Pernambuco (Farr 1960, Cavalcanti 1976, Pôrto 1982), Paraíba (Cavalcanti \& Araújo 1985, Cavalcanti \& Marinho 1985, Cavalcanti \& Oliveira 1985, Cavalcanti \& Silva 1985) e Maranhão (Torrend 1915). Até o momento, não se tem registros para Ceará, Piauí, Rio Grande do Norte e Sergipe.

Nos estados do Nordeste estes organismos são encontrados tanto em ecossistemas naturais, como caatingas, matas serranas e matas úmidas, como sobre plantas cultivadas de interesse ornamental ou econômico (Cavalcanti et al. 1985). Santos (1988), em seu trabalho sobre Myxomycetes encontrados em cana-de-açúcar, relaciona várias espécies de vegetais superiores como, aveia, café, cevada, fumo, trigo, também propícias ao desenvolvimento e frutificação desses organismos. Pôrto et al. (1985), estudando a ocorrência de Myxomycetes em quatro espécies de palmeiras, em Pernambuco (Acrocomia intumescens Drude, Cocos nucifera L. , Elaeis guineensis L., Mauritia vinifera Mart. ), relataram 23 espécies, distribuídas em 19 gêneros, sobre diferentes órgãos, vivos ou em decomposição.

As palmeiras encontradas na Chapada do Araripe, Sul do Estado do Ceará, pertencem principalmente às espécies Acrocomia intumescens Drude (macaúba), Copernicea prunifera (Mill.) H. E. Moore (carnaúba), Mauritia flexuosa Mart. (buriti) e Orbygnia phalerata Mart. (babaçu). O babaçu, a macaúba e o buriti, com predominância do primeiro, apresentam maiores populações no trecho beneficiado pelas ressurgências, 500-600 m de altitude, onde são encontradas em faixas mais densas, rareando para cotas altitudinais inferiores. O buriti é mais freqüente nos locais brejosos, daí ser localmente conhecido como "palmeira dos brejos" (Bastos \& Figueiredo 1987).

Visando contribuir para o melhor conhecimento das áreas de ocorrência dos Myxomycetes no Brasil, particularmente no Nordeste, bem como dos substratos que lhes são propícios para frutificação, foi realizado um levantamento das espécies que são encontradas sobre palmeiras, no Município do Crato, Ceará.

\section{Material e métodos}

Área de coleta

$\mathrm{O}$ material estudado foi proveniente de coletas aleatórias efetuadas em cinco excursões no Estado do Ceará, Microrregião do Cariri ( $7^{\circ} 30^{\prime} 00^{\prime \prime} \mathrm{S}, 39^{\circ} 00^{\prime} 00^{\prime \prime} \mathrm{W}$, 400-120 m de altitude), Município do Crato (Figura 1). 


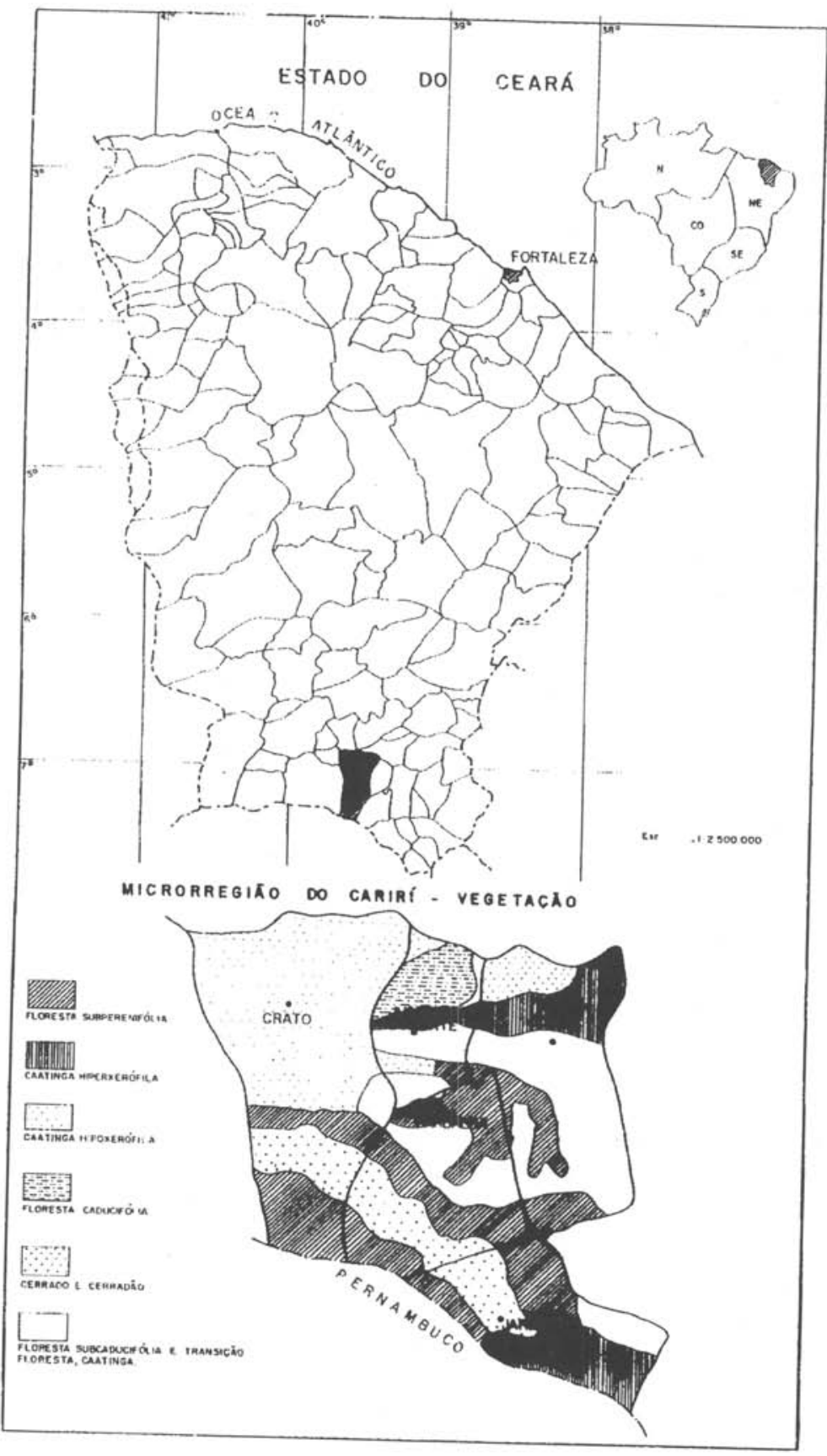

Figura 1. Localização da área de coleta de Myxomycetes (Crato, Microrregião do Cariri, CE) 
A Região é dominada pela Chapada do Araripe, que forma um conjunto tabular com as encostas voltadas para o Sul e Sudeste, tornando o relevo o principal elemento do quadro natural. O clima da Microrregião do Cariri caracteriza-se por apresentar temperaturas amenas e chuvas orográficas, decorrentes da localização da Chapada, que provoca precipitações abundantes e alta umidade (SUDEC, 1972).

A vegetação local é muito diversificada em função do clima e relevo; no topo da Chapada encontra-se vegetação do tipo floresta tropical subperenifolia; na encosta, cerrado e cerradão e nas áreas circunvizinhas, de relevo ondulado, caatinga hipoxerófila e floresta tropical caducifólia (Figura 1). A permeabilidade das rochas sedimentares permite a ressurgência das águas de infiltração e essas nascentes dão origem aos brejos nas meias encostas. Nestes locais, geralmente às margens dos riachos e proximidades, foram realizadas as coletas, no final da estação chuvosa.

\section{Coleta, herborização e identificação}

Investigou-se a presença de Myxomycetes em quatro espécies de palmeiras nativas na região (Acrocomia intumescens, Copernicea prunifera, Mauritia flexuosa e Orbygnia phalerata), examinando-se diferentes órgãos de exemplares vivos ou em decomposição.

A coleta e acondicionamento do material foram realizados segundo a metodologia indicada por Cavalcanti (1974). No laboratório, o material foi colocado na estufa a $36^{\circ} \mathrm{C}$, para secagem e posterior herborização.

Para análise dos corpos frutíferos adotou-se a metodologia descrita por Farr (1976). Para identificação dos espécimes utilizou-se Lister (1925), Martin \& Alexopoulos (1969) e Farr (1976).

As exsicatas dos espécimes estudados encontram-se depositadas no Herbário UFP, do Departamento de Botânica, Centro de Ciências Biológicas, Universidade Federal de Pernambuco.

\section{Resultados e Discussão}

Foram observadas frutificações de Myxomycetes em indivíduos pertencentes às quatro espécies de palmeiras selecionadas, exclusivamente sobre órgãos mortos, em decomposição. Verificou-se a presença de representantes das três subclasses, destacando-se, pela diversidade de espécies, os Myxogastromycetidae (Tabela 1).

Dentre as espécies relatadas para Pernambuco por Pôrto et al. (1985), apenas $23,4 \%$ foram encontradas sobre órgãos vivos (estipe ou folhas), abrangendo os gêneros Badhamia, Physarum, Fuligo, Didymium, Diderma, Arcyria, Hemitrichia e Cribraria. Gill \& Onyibe (1986), no Oeste da África, pesquisando epífitas sobre Elaeis guineensis, assinalaram a presença de Myxomycetes em exemplares vivos dessa palmeira, porém numa baixa freqüência $(10 \%)$ e pequena diversidade de gêneros (Physarum e Stemonitis), distribuídos em três espécies. Todos esses gêneros, com exceção de Diderma e Didymium, estão presentes no material analisado, apesar de não terem sido encontrados frutificando sobre indivíduos vivos (Tabela 1). 
Tabela 1. Myxomycetes assinalados sobre diferentes órgãos de palmeiras ocorrentes no Município do Crato-CE.

\begin{tabular}{|c|c|c|c|}
\hline \multirow{2}{*}{ TÁxONS } & \multicolumn{3}{|c|}{ SUBSTRATO } \\
\hline & Espata & Folha & Estipe \\
\hline \multicolumn{4}{|l|}{ Ceratiomyxaceae } \\
\hline Ceratiomyxa fruticulosa (Müll.) Macbr. & + & $\cdot$ & $\cdot$ \\
\hline \multicolumn{4}{|l|}{ Cribrariaceae } \\
\hline $\begin{array}{l}\text { Dictydium cancellatum (Batsch) Macbr. } \\
\text { Trichiaceae }\end{array}$ & - & $\cdot$ & + \\
\hline Arcyria cf. magna Rex & \\
\hline Hemitrichia calyculata (Speg.) Farr & 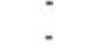 & $\begin{array}{l}+ \\
+\end{array}$ & 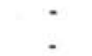 \\
\hline \multicolumn{4}{|l|}{ Physaraceae } \\
\hline Badhamia affinis Rost. & + & - & - \\
\hline Fuligo septica (L.) Wigg. & - & - & + \\
\hline Physarum nucleatum Rex & - & - & + \\
\hline Physarum stellatum (Mass.) Mart. & - & - & + \\
\hline Physarum sp. & - & - & + \\
\hline \multicolumn{4}{|l|}{ Stemonitaceae } \\
\hline Comatricha typhoides (Bull.) Rost. & - & - & + \\
\hline Stemonitis pallida Win. & - & + & + \\
\hline Stemonitis sp. & $\cdot$ & - & + \\
\hline
\end{tabular}

Comparando-se a mixomicota relacionada às quatro espécies de palmeiras aqui estudadas com as referidas para Pernambuco por Pôrto et al. (1985), observou-se 50\% de semelhança a nível de espécies, evidenciando-se que os Myxomycetes não são específicos para determinada forófita, desde que os dois levantamentos incluem diferentes espécies de palmeiras. Além disso, estes organismos parecem ser indiferentes à natureza do órgão e estado em que o mesmo se encontra, pois apenas Dictydium cancellatum e Physarum nucleatum coincidem nas duas listagens quanto ao tipo e estado do órgão em que frutificaram. Hemitrichia calyculata e Arcyria magna, referidas por Pôrto et al. (1985) para estipe morto, foram encontradas no Crato sobre folhas mortas; Fuligo septica, antes referida para folhas vivas ou em decomposição, foi agora assinalada sobre estipe morto e Badhamia affinis, anteriormente relatada para estipe vivo, foi aqui registrada sobre espata morta. Na mixomicota encontrada nas palmeiras analisadas no Crato não estão incluídas as quatro espécies que tiveram maior número de assinalamentos para este substrato em Pernambuco (Pôrto et al. 1985). No levantamento efetuado, acrescenta-se à lista dos Myxomycetes que frutificam sobre palmeiras um gênero (Ceratiomyxa) e quatro espécies (Ceratiomyxa fruticulosa, Comatricha typhoides, Stemonitis pallida e Physarum stellatum), todos com ampla distribuição mundial e referidos para diversos tipos de substratos (Martin \& Alexopoulos 1969, Farr 1976).

Com o presente trabalho, eleva-se para 35 o número de espécies de Myxomycetes que ocorrem em palmeiras, tornando-se evidente que estas monocotiledôneas, 
representadas por seis gêneros, constituem substrato propício ao desenvolvimento e frutificação desses organismos.

\section{Agradecimento}

As autoras agradecem à Pró-reitoria Comunitária da Universidade Federal de Pernambuco, na pessoa do Prof. Affonso Barros de Almeida e à Universidade Regional do Cariri, nas pessoas da Prof. ${ }^{a}$ Terezinha Gonçalves Batista e do Prof. Francisco A. B. da Cunha, pelo apoio dispensado para a viagem e excursões para coleta.

\section{Referências bibliográficas}

Bastos, A. P. P. \& Figueiredo, M. A. 1987. O nível das palmáceas na Chapada do Araripe, CE. In, Reunião Nordestina de Botânica IX, Fortaleza, Universidade Federal do Ceará, p. 29.

Cavalcanti, L. H. 1974. Mixomicetos corticícolas do cerrado de Emas (Pirassununga-São Paulo). Dissertação de Mestrado, Instituto de Biociências: Universidade de São Paulo.

Cavalcanti, L. H. 1976. Mixomicetos novos para Pernambuco II. Universidade Federal de Pernambuco, Ciências Biológicas, Recife. Série Botânica, 4 (4): 1-19.

Cavalcanti, L. H. \& Araújo, V. L. F. 1985. Myxomycetes da Paraíba II — Liceales. In: Anais da VIII Reunião Nordestina de Botânica. Recife, Sociedade Botânica do Brasil, Seccional Pernambuco, p. 193-198.

Cavalcanti, L. H. \& Marinho, M. G. V. 1985. Myxomycetes da Paraíba I - Trichiales. In: Anais da VIII Reunião Nordestina de Botânica. Recife, Sociedade Botânica do Brasil, Seccional Pernambuco, p. 185-191.

Cavalcanti, L. H. \& Oliveira, I. C. 1985. Myxomycetes da Paraíba IV - Stemonitales. In: Anais da VIII Reunião Nordestina de Botânica. Recife: Sociedade Botânica do Brasil, Seccional Pernambuco, p. 207-214.

Cavalcanti, L. H. \& Silva, J. V. B. 1985. Myxomycetes da Paraíba III - Physarales. In: Anais da VIII Reunião Nordestina de Botânica. Recife; Sociedade Botânica do Brasil, Seccional Pernambuco, p. 199-205.

Cavalcanti, L. H.; Santos, E. J.; Silva, M. I. L. \& Pinto, I. M. A. 1985. Myxomycetes em cana-de-açúcar (Saccharum officinarum L.). In: Anais da VIII Reunião Nordestina de Botânica. Recife: Sociedade Botânica do Brasil, Seccional Pernambuco, p. 215-221.

Farr, M. L. 1960. The Myxomycetes of the IMUR herbarium, with special reference to Brazillian species. Recife, Instituto de Micologia, 184: 1-54.

Farr, M. L. 1976. Myxomycetes. Flora neotropica. New York: New York Botanical Garden. (Monograph n. $\left.{ }^{\circ} 16\right)$

Gill, L. S. \& Onyibe, H. I. 1986. Phytosociological studies of epiphytic flora of oil palm (Elaeis guineensis Jacq.) in Benin City, Nigéria. Feddes Repertorium 97 (9-10): 691-695.

Gottsberger, G. 1968. Myxomyceten aus Bahia und Goiás. Nova Hedwigia 15: 316-368.

Lister, A. 1925. A monograph of the Mycetozoa. London: British Museum Natural History,

Martin, G. W. \& Alexopoulos, C. J. 1969. The Myxomycetes. Iowa City: University of Iowa Press.

Pôrto, K. C. 1982. Myxomycetes da mata de Dois Irmãos, Recife - Pernambuco: Dissertação de Mestrado. Universidade Federal Rural de Pernambuco.

Pôrto, K. C.; Cavalcanti, L. H. \& Correia, A. M. S. 1985. Incidência de Myxomycetes em Palmae. In: Anais do XXXIII Congresso Nacional de Botânica. Brasília; EMBRAPA, p. 181-187.

Santos. E. J. 1988. Sistemática e ecologia de Myxomycetes ocorrentes em cana-de-açúcar (Saccharum sp). Dissertação de Mestrado. Recife: Universidade Federal de Pernambuco. 
Santos. E. J. \& Cavalcanti. L. H. , Albuquerque, W. C. 1986. Myxomycetes de Alagoas. In: Anais do XXXIII Congresso Nacional de Botânica. Anais. Ouro Preto: Universidade Federal de Ouro Preto; p. 499-504. SUDEC. 1972. A microrregião plana do Cariri. Fortaleza: Convênio SUDENE/ Estado do Ceará /
SERFHAU.

Torrend, C. 1915. Les Myxomycetes du Brasil, Connus jusquici. Broteria. Série Botânica 13: 72-88. 